\title{
AL MARGEN DEL RECONOCIMIENTO:
} MANIFESTACIONES DEL HETEROSEXISMO, LA HOMOFOBIA, LA LESBOFOBIA Y LA BIFOBIA EN LA EDUCACIÓN DEL TRABAJO SOCIAL 


\section{Odalis Reyes Prado}

Tiene Bachillerato en Trabajo Social de la Universidad de Puerto Rico, Recinto de Río Piedras, y Maestría en Trabajo Social de la Escuela Graduada de Trabajo Social Beatriz Lasalle de la Universidad de Puerto Rico, Recinto de Río Piedras. Realizó múltiples prácticas profesionales donde intervino con personas de edad avanzada, estudiantes de escuela elemental e intermedia, y con mujeres y niñas sobrevivientes de agresión sexual. Trabaja en una organización no gubernamental sin fines de lucro dirigida a mujeres sobrevivientes de violencia de género, donde provee ayuda psicosocial, talleres educativos y grupos de apoyo, desde una perspectiva feminista y de género.

\section{José Miguel Ayala Salamán}

Posee un Bachillerato en Trabajo Social de la Universidad de Puerto Rico, Recinto de Río Piedras. Culminó en el 2014 su Maestría en Trabajo Social de la Escuela Graduada de Trabajo Social Beatriz Lasalle de la Universidad de Puerto Rico, Recinto de Río Piedras. Durante sus estudios universitarios, formó parte de múltiples organizaciones estudiantiles. Colaboró, como parte de sus prácticas profesionales, con el Departamento de Consejería para el Desarrollo Estudiantil de la Universidad de Puerto Rico, Recinto de Río Piedras (DCODE), que brinda servicios de psicología, trabajo social y consejería a estudiantes activos/as del Recinto.

\section{José Antonio Hernández Olavarría}

Tiene un Bachillerato en Trabajo Social de la Universidad de Puerto Rico, Recinto de Río Piedras, y Maestría en Trabajo Social de la Escuela Graduada de Trabajo Social Beatriz Lasalle. Realizó sus prácticas profesionales en el Centro de Autismo de Puerto Rico del Instituto de Deficiencias en el Desarrollo de la Universidad de Puerto Rico y en la Escuela Intermedia José Celso Barbosa, en Río Piedras.

Recibido:

18 de mayo de 2014

Aprobado:

7 de octubre de 2014 


\section{AL MARGEN DEL RECONOCIMIENTO: MANIFESTACIONES DEL HETEROSEXISMO, LA HOMOFOBIA, LA LESBOFOBIA Y LA BIFOBIA EN LA EDUCACIÓN DEL TRABAJO SOCIAL}

Odalys Reyes Padró, José M. Ayala Salamán y José A. Hernández Olavarría

Resumen

En este artículo se presenta el análisis de una investigación cualitativa que exploró las manifestaciones del heterosexismo, la homofobia, la lesbofobia y la bifobia en la educación de Trabajo Social a nivel de bachillerato en la Universidad de Puerto Rico, Recinto de Río Piedras, con el ánimo de provocar la discusión y reflexión de la educación de Trabajo Social. Era crucial evaluar el proceso de enseñanza de Trabajo Social para promover una transformación en la educación.

Como parte de los hallazgos de la investigación se encontró que profesoras, profesores y estudiantes que participaron en la investigación mostraron dificultad para definir conceptos relacionados a la diversidad sexual, y hubo poca o ninguna inclusión del tema en las clases observadas y en los temarios. Los hallazgos fueron analizados 
desde el construccionismo social, la teoría crítica, la teoría de socialización y la perspectiva de género. Esto permitió explicar cómo la sexualidad ha sido construida socialmente desde el patriarcado, el machismo y el heterosexismo, y se ha rechazado cualquier otra manifestación de la sexualidad, de manera que la heteronormativa se ha legitimado a través de los procesos de socialización. Por tanto, como parte de la reflexión, fue importante entender que resulta complejo desprenderse de los prejuicios y la homofobia/lesbofobia/bifobia. Por esa razón se promovió el cuestionamiento a las normativas y a las instituciones sociales, para provocar la deconstrucción de ideas opresivas. Los hallazgos invitan a reflexionar críticamente la educación que se recibe y hacia dónde nos dirigimos como profesionales que aspiramos a una sociedad justa.

Palabras claves: homofobia, lesbofobia, bifobia, heterosexismo, diversidad sexual, Trabajo Social, educación.

\section{Abstract}

This articlepresentstheanalysis ofaqualitativeresearch that explored the manifestations of heterosexism, homophobia, lesbophobia and biphobia in social work education at the undergraduate level at the University of Puerto Rico, Río Piedras campus. The findings are presented in order to provoke a reflective discussion on the practice of social work education.

One significant finding showed that professors and 
students exhibited difficulty in defining concepts related to sexual diversity. Moreover, there was little or no inclusion at all of the topic in the classes observed and the curricula verified. The findings were analyzed from the perspectives of social construction theory, critical theory, socialization theory and gender perspective theory. This holistic approach enabled us to explain how society has defined the sexuality from patriarchy, male chauvinism and heterosexism, and rejected any other manifestation of sexuality and gender. That is, heteronormativity has been legitimized through socialization processes and thereafter replicated by the people, in this case Social Work educators and students. Therefore, as part of deliberation generated, it was important to understand the difficulty and effort required to get rid of prejudice, stereotypes and homophobia/ lesbophobia/ biphobia. For that reason, the study presents testimonies which indubitably promote critical questioning of social norms, traditions and social institutions as it promotes the deconstruction of oppressive thoughts. The findings lead to a critical reflection on the education received and illustrates where we should be headed as professionals who seek equity and a more fair society.

Keywords: homophobia, lesbophobia, biphobia, heterosexism, sexual diversity, Social Work, education. 


\section{Introducción}

¿Es heterosexista, homofóbica, lesbofóbica y/o bifóbica la educación, a nivel de bachillerato de Trabajo Social en la Universidad de Puerto Rico, Recinto de Río Piedras? Esta pregunta pareciera tener una respuesta obvia: ¡No! Esto porque se comenta que en la Universidad del Estado existe un ambiente de inclusión y aceptación a la diversidad. Sin embargo, si bien es cierto que existe mayor acogida a la diversidad de ideas, la Universidad es parte de una sociedad que se rige por normas sociales que influyen en cómo se practica la docencia.

Esa interrogante dirigió la investigación cualitativa realizada en la Universidad de Puerto Rico de la cual se hace mención en este artículo, y en la cual participaron cinco profesores y profesoras. A estos(as) se les realizó una entrevista semiestructurada y se llevaron a cabo observaciones pasivas en una de sus clases. De igual manera se analizó el contenido de los sílabos de las clases observadas, todo con la intención de describir, analizar y constatar la información resultante. En contraparte, se realizaron entrevistas semi-estructuradas a profundidad a tres estudiantes que se identificaron como homosexuales, lesbianas, bisexuales o heterosexuales, y que cursaban su tercer año o años superiores en el Programa de Bachillerato de Trabajo Social de la Universidad de Puerto Rico, Recinto de Río Piedras, con el objetivo de comparar los discursos del profesorado con los discursos del estudiantado. Los hallazgos fueron analizados desde un marco conceptual que integró elementos de la teoría del construccionismo social, la teoría crítica y la teoría de socialización, todos enmarcados desde la perspectiva de género. De igual modo se utilizó la técnica de triangulación para comparar y contrastar los hallazgos. 
El objetivo principal de este artículo es ofrecer un resumen de la investigación realizada, poniendo énfasis en el análisis y la triangulación formulada, ya que promueven la discusión sobre la importancia de revisar la educación en Trabajo Social para atemperarla a la realidad social.

\section{Planteamiento del problema}

La intervención del/la profesional de Trabajo Social se justifica cuando sus prácticas van dirigidas a fomentar los derechos humanos, la diversidad, la equidad, la justicia y la participación activa y crítica de los participantes en la solución de problemas o necesidades (Colegio de Profesionales del Trabajo Social, 2010, p.12).

Cualquier persona que lea este código de ética entraría, probablemente, en un estado de éxtasis y regocijo al leer un documento que, a simple vista, aparenta ser tan progresista que fácilmente podría ser un estandarte de justicia social. Es tan convincente en sus postulados de equidad, que la primera imagen que llega a la mente del lector o lectora del documento es la de profesionales del Trabajo Social libres de prejuicios, estereotipos y opresiones o encaminados(as) hacia ello.

No obstante, se ha evidenciado que la realidad del o la profesional del Trabajo Social es distinta. "Ello se debe a que estos(as) comparten inconscientemente los prejuicios heterosexistas de la sociedad y, además, tienen un déficit de información sobre la homosexualidad que les permita interactuar efectivamente con esta población" (Nieves, 2011, pp.17-18). Igualmente debemos mencionar que los(as) educadores(as) de Trabajo Social no están exentos(as) de 
esta realidad, pues son seres socializados(as) con los mismos valores que las demás personas de la sociedad y, en algunos casos, pueden desconocer el tema de la diversidad sexual. De esta manera, quienes son profesores(as) o maestros(as), podrían transmitir, a través de la cátedra, estos discursos heterosexistas, homofóbicos, lesbofóbicos y bifóbicos a los(as) estudiantes, en algunos casos de manera consciente, pero sin admitir sus prejuicios, y en otros de modo inconsciente, sin darse cuenta del prejuicio emitido.

\section{Marco conceptual}

El marco conceptual integra elementos de la teoría del construccionismo social, la teoría de socialización y la teoría crítica, que aportan información útil y valiosa acerca del proceso de aprendizaje, modelaje y reproducción de las fobias mencionadas, y se ofrece una posible explicación coherente sobre cómo las personas lesbianas, homosexuales y bisexuales (LHB) asumen los discursos opresivos provocando que, incluso, los internalicen y emulen. Todos los conceptos teóricos utilizados son abordados desde la perspectiva de género (que articula en su interior los elementos de las diferentes fuentes teóricas mencionadas) que, según Lamas (1995), es una categoría analítica que permite cuestionar todas las construcciones sociales, culturales y políticas que tienen repercusiones en las desigualdades entre hombres y mujeres por cuestiones de roles de género, identidades de género y diversidad sexual.

La teoría del construccionismo social fue ideal para realizar acercamientos al tema de estudio, debido a que el construccionismo social comprende que la realidad es construida socialmente, y a su vez "...es lenguaje elaborado 
en interacción social y no existe una verdad. La verdad es múltiple y arraigada en el contexto socio-histórico y cultural en que se construye" (Albite, 2004, p.2). Además, según Kisnerman (2005), la realidad se construye mediante las interacciones y el diálogo entre las personas, por lo que no hay verdades absolutas. Es mediante esas interacciones que las personas construyen significados y simbolismos que interpretan de acuerdo a su propia experiencia, subjetividad y visión de mundo. Estos postulados teóricos permiten retar la verdad absoluta y las normativas aceptadas socialmente como la realidad y, por lo tanto, promueven el cuestionamiento a la heteronormativa que está cobijada por las construcciones androcéntricas, machistas y patriarcales de la sexualidad.

Hacia esa dirección de duda y escepticismo es que nos conduce la teoría crítica que nos propone Connerton (1976), según citado por Ritzer (2002), donde se expone que la realidad social se debe cuestionar, desenmascarar y develar. Para promover el pensamiento crítico se parte de un cuestionamiento de lo establecido y, al integrar un enfoque desde la perspectiva de género, se puede cuestionar la heterosexualidad compulsoria y la construcción del género y la sexualidad.

A la sociedad no le interesa reconocer una línea de pensamiento distinta a la que permea y mucho menos le interesa enseñar otra línea de pensamiento a la que ya está impuesta, sino pretende reproducir lo establecido sin retarlo o cuestionarlo. Por lo tanto, se busca reproducir y reforzar la normativa que promueve la desigualdad entre los géneros y las sexualidades debido a que:

"Los discursos de legitimación de género se hallan presentes en casi todas las formas culturales: los mitos, las grandes religiones, la filosofía, la literatura, 
los medios, las ciencias sociales y, en ocasiones, las ciencias naturales. De manera directa o encubierta, dan una explicación de la desigualdad entre los sexos tendentes a su conservación." (Puleo, 2005, p.23).

Este proceso de legitimación imposibilita o dificulta otras visiones diversas, justas y libertarias sobre la diversidad sexual y de los géneros.

Estos discursos también se legitiman a través del proceso de socialización, en el cual las personas interactúan con la sociedad y su medio ambiente intercambiando, internalizando y reproduciendo aspectos sociales, culturales e históricos. "La socialización es el proceso por el cual la criatura indefensa se va convirtiendo gradualmente en una persona consciente de símisma, con conocimientos y diestra en las manifestaciones de la cultura en la que ha nacido." (Giddens, 2000, p. 51) Sin embargo, esta cultura responde a construcciones patriarcales y, por ende, machistas y androcéntricas, donde se establece una división desigual y polarizada de los géneros.

Esto significa que las personas son socializadas en entornos patriarcales y machistas, por tanto tienden a ser heterosexistas, lesbofóbicas, homofóbicas y bifóbicas. Por eso "... resulta claro que las orientaciones que un actor instrumenta en el curso de su interacción complementaria en los roles no son innatas, sino que han de adquirirse a través de un aprendizaje" (Parsons, 1951, p.134). Las personas no nacen siendo homofóbicas, lesbofóbicas y bifóbicas. Lo aprenden de otras personas mientras crecen y son educados(as), o sea, socializados(as). Estos discursos son internalizados, reproducidos y reforzados, usualmente de manera inconsciente, por parte de los(as) socializados(as) que funcionan, entonces, como entes disgregativos de esas construcciones sociales que son aprendidas y acatadas por los(as) receptores porque 
no se tienen elementos para contradecir las mismas. Ese conocimiento adquirido pasa de generación en generación y se esparce como un virus que se "contagia" y se mantiene. De esa manera se naturaliza y se transforma en una normativa que se inserta en la tradición y la cultura, de modo que no se cuestiona porque se da y se toma como un hecho histórico insoslayable.

\section{Metodología del estudio}

En la investigación se realizó un estudio de campo, en el cual se exploró las manifestaciones del heterosexismo, la lesbofobia, la homofobia y la bifobia en la educación de Trabajo Social desde la perspectiva de los(as) profesores(as) y de los(as) estudiantes del Programa de Bachillerato en Trabajo Social de la Universidad de Puerto Rico, Recinto de Río Piedras. Se utilizó una metodología cualitativa con la intención de describir y analizar la información resultante mediante una estrategia multi-modal de entrevistas semi-estructuradas, observaciones pasivas y análisis de contenido. Se llevaron a cabo entrevistas semi-estructuradas a profundidad a cinco profesoras y profesores de Trabajo Social que ofrecían cursos medulares. De igual manera, se entrevistó a tres estudiantes de Trabajo Social a nivel sub-graduado que estuviesen cursando su tercer año académico o años superiores y se identificaran como homosexuales, heterosexuales, lesbianas o bisexuales. Además, se realizaron observaciones de algunas de las clases que imparten los(as) profesores(as) que participaron del estudio. También se revisaron y analizaron los sílabos de las clases observadas. Esto permitió realizar un análisis comparativo de la información resultante, ya que se pudo ver la perspectiva que tienen los(as) profesores(as) y la perspectiva de los(as) estudiantes. 


\section{Análisis de los hallazgos}

Los hallazgos del estudio coincidieron con la literatura revisada, ya que develaron cómo se están manifestando el heterosexismo, la homofobia, la lesbofobia y la bifobia en los salones de clases, los currículos y en los discursos emitidos por el profesorado y el estudiantado de Trabajo Social que participó de la investigación. El análisis de los hallazgos se realizó partiendo de las entrevistas semi-estructuradas que se efectuaron con los(as) profesores(as) del Bachillerato en Trabajo Social. Este análisis no pretende generalizar, sino ofrecer posibles explicaciones desde las narrativas y discursos ofrecidos por los(as) participantes del estudio. Sus testimonios y discursos fueron interpretados desde el marco conceptual adoptado.

Como parte de las entrevistas, se realizaron preguntas abiertas para explorar sus opiniones sobre diversidad sexual, para luego poder analizar los discursos. Algunos de los temas abordados en las preguntas estuvieron enfocados en conocimientos de conceptos, abordajes en el salón de clases, en el currículo, el programa de Trabajo Social y en la sociedad en general. Una de las preguntas giró en torno a la inclusión de los cursos medulares en cuanto al tema de diversidad sexual. La opinión de los(as) profesores(as) fue diversa, pero se inclinaron a que se trabaja el tema por imposición de la agencia acreditadora. En este contexto, los testimonios ofrecidos evidenciaron las diferencias individuales en el manejo y abordaje de la temática de diversidad sexual entre el profesorado. En este sentido se puede entender que, dependiendo de los intereses de cada profesor o profesora y de las experiencias profesionales y personales, fue la manera en que se trabajó el tema en cuanto a frecuencia y profundidad. Mientras se manifestaron diferentes realidades de lo que sucede en las 
aulas, quedó claro que existe una corriente dominante que tiende a trabajar marginalmente el tema de la diversidad sexual.

En respuesta a la pregunta sobre si la temática de diversidad sexual se aborda en la profesión de Trabajo Social, la mayoría coincidió en que no se aborda. Para los(as) entrevistados(as), la profesión de Trabajo Social en Puerto Rico no se ha inmerso ampliamente en la discusión de la temática. Estas respuestas se pueden visualizar de dos formas: la primera, desde la teoría crítica, la cual propone cuestionar la normativa dominante para trastocar su legitimidad; la segunda, desde el construccionismo social y la socialización, las respuestas cobran un matiz particular. Esto se debe a que el problema yace en que los(as) entrevistados(as) han internalizado los discursos dominantes de la sexualidad y aún no se han dado a la tarea de cuestionar sus creencias y valores como profesionales. La profesión de Trabajo Social tiene una ética y unos valores de respeto a la diversidad, de justicia social y de inclusión. Sin embargo, quienes la practican o la enseñan son quienes tienen que comenzar por deconstruir y cuestionar lo asumido e internalizado a través del proceso de socialización.

Al explorarse si consideraban al Trabajo Social como homofóbico, lesbofóbico y/o bifóbico, la mayoría se inclinó por aclarar que la profesión no lo es, pero sí algunos trabajadores(as) sociales. Por ejemplo, una persona del profesorado manifestó: "...la profesión no es homofóbica, los que son más homofóbicos somos los que estamos dentro de la profesión". Para mostrar la complejidad de lo expuesto, cabe destacar que la mayoría de los profesores y las profesoras consideraron que el heterosexismo, la homofobia, la lesbofobia y la bifobia no son lo mismo, pero tienen las mismas intenciones. Sobre esto una persona del profesorado expresó: 
“...yo no definiría igual los términos, pero definitivamente si yo soy heterosexista y yo me paro en esa posición, y nada más existe, estoy entrando, cruzando esa línea así como, verdad, tan finita y casi invisible a la homofobia porque yo estoy de facto excluyendo hasta una visión".

Al explorar si la educación del programa de Trabajo Social incluye la temática de diversidad sexual, las respuestas estuvieron dispersas, aunque se pueden vincular y relacionar. Una de las personas del profesorado manifestó:

"...el currículo no pretende eso, o sea, si el currículo no pretende eso, no te va a preparar para eso. El currículo sí puede moverte a que tú tengas aceptación hacia la diversidad, pero la diversidad es muy amplia... De hecho, no hay centros de práctica para intervenir con comunidades LGBTT, a saber no están, no los buscan, y existen, pero no se ubican estudiantes..."

Ante lo anterior expuesto, es imperante presentar un análisis crítico que no pretende generalizar, sino ofrecer posibles explicaciones de los hallazgos obtenidos de las entrevistas semi-estructuradas realizadas a estudiantes de bachillerato en Trabajo Social participantes del estudio. Se exploró, mediante preguntas abiertas enfocadas en el conocimiento de conceptos, abordaje del tema en las clases, currículos, el Programa de Trabajo Social, y su sentir como estudiantes lesbianas, homosexuales, bisexuales o heterosexuales en la academia. Se auscultó si la temática de diversidad sexual y de género en los salones de clase aborda la inclusión de la temática LHB. Los(as) estudiantes coincidieron en que el tema no se aborda, aunque existen ciertas excepciones en algunas clases en específico. Por ejemplo, una persona del estudiantado indicó: 
"Eeeh, sinceramente considero que no, con algunas excepciones en profesores o algunos cursos en específico. Pues si da pie para crear como uno, dice la curiosidad en una persona de buscar más allá en el tema. Pero la realidad es que pienso que a nivel, eeh, académico y teórico, eeh, el currículum no va dirigido necesariamente a... a crear esa conciencia a nivel del estudiantado. Sí hay excepciones de profesores, repito $y$ de clases, pero en general me parece que puede ser un poco evasivo en ese sentido".

Definitivamente, los(as) estudiantes reconocen que el tema no es trabajado con amplitud y que el abordaje al mismo varía según el(la) profesor(a) y el curso. No obstante, ellos(as) hicieron hincapié en la importancia de incorporar el tema para que se trabaje con amplitud, frecuencia y profundidad.

Al explorar la opinión de los(as) estudiantes en cuanto a la frecuencia del uso de ejemplos relacionados a la temática de diversidad sexual, por un lado indicaron que se aborda por interés del estudiantado, y por otro consideran que se utilizan pocos ejemplos, pero con algunas excepciones.

De este mismo modo, una persona del estudiantado manifestó:

"...bien poco bien poco, en los cursos medulares, si acaso yo oí, bien poco durante los cursos medulares sí se hace referencia, se puede hacer referencia una vez a la clase, en la clase, pero no es algo que es común, no es algo que es ejemplo".

Esto refleja que los(as) estudiantes son conscientes del sesgo heterosexista en la educación, y que al menos ellos(as) están en la apertura de profundizar críticamente en aspectos como es la diversidad sexual. 
Se auscultó si los(as) estudiantes pensaban que el Trabajo Social era heterosexista. Una persona contestó que sí es heterosexista y que ello se evidenciaba por la población a la cual se dirigen los servicios en Puerto Rico. El resto dijo que el Trabajo Social es heterosexista por el contexto social en el cual está insertada la profesión. Una persona del estudiantado verbalizó:

"Definitivamente este, yo creo que no podemos excluir a la profesión de una cultura y una sociedad en que... es totalmente patriarcal y heterosexista. O sea es una profesión aunque, si considero que la generación... tenemos una generación de profesionales y de futuros profesionales que vienen con una idea un poco distintas, con una ideas que van... con una ideas transformadoras frente al tema".

Con esa respuesta, él o la estudiante cuestiona el contexto social que impacta la profesión de Trabajo Social y las agencias donde se ejerce la misma. De igual manera, hace alusión a una nueva generación de profesionales con ideas distintas e inclusivas. Ese comentario parece apuntar a que hay una generación que lleva años ejerciendo la profesión desde las ideologías machistas, patriarcales y heterosexistas. Por lo tanto, estos(as) estudiantes se posicionan a sí mismos(as) como una nueva generación que está dispuesta a deconstruir y reflexionar críticamente los asuntos relacionados a las personas LHB.

De este mismo modo, se investigó la opinión de los(as) estudiantes en cuanto a si el Trabajo Social es homofóbico, lesbofóbico y/o bifóbico. La tendencia en las respuestas fue a aclarar que la profesión no es homofóbica, lesbofóbica ni bifóbica, sino que algunos(as) profesionales lo son. Las 
reacciones a esta pregunta fueron bastante parecidas a las que tuvieron los(as) profesores, de manera que se entiende que lo expresado es una contradicción si se toma en cuenta la respuesta que dieron a la pregunta sobre si la profesión de Trabajo Social es heterosexista. Si partimos de la premisa de que la homofobia, la bifobia y la lesbofobia son manifestaciones que se derivan del heterosexismo y que tienen su raíz en el patriarcado, resulta ilógico en cierta manera argumentar que la profesión es heterosexista, pero no es LHBfóbica. De hecho, los(as) entrevistados(as) mismos encontraron relación entre los conceptos. Por ejemplo, una persona del estudiantado indicó:

“...son una producto de otra. Yo creo que la homofobia, la lesbofobia, etc. Son producto de una ideología patriarcal heterosexista. Está intrínsecamente relacionado, pero no son lo mismo definitivamente. El heterosexismo va directamente a plantear con las categorizaciones de género a nivel social, a nivel del poder, en todos los aspectos. Ya sea desde el poder político, profesión, hasta lo que es el hogar y los procesos de desarrollo de la familia etc. Y a raíz de esto obviamente y en lo que es la homofobia o la lesbofobia que ya va... vemos que es producto, no, de esas ideologías divisoras del heterosexismo".

Por lo tanto, si la profesión es heterosexista, es decir, que legitima la heterosexualidad compulsoria, es consecuente decir también que es homofóbica, lesbofóbica y bifóbica, debido a que el heterosexismo desplaza cualquier otra variante de la sexualidad.

Por último, se auscultó la percepción de los(as) estudiantes en cuanto a si el Programa de Bachillerato en Trabajo Social prepara futuros(as) trabajadores(as) sociales para intervenir 
con personas LHB. Las respuestas fueron diversas, por lo que no coincidieron. Hubo una persona que expresó que el Programa no prepara a los(as) y los(as) futuros(as) trabajadores(as) sociales de la manera adecuada para intervenir con la población LHB. Otra persona indicó que el Programa sí prepara adecuadamente, y la tercera persona informó que todo dependía, por lo que algunas veces el Programa sí preparaba y otras veces no.

\section{Triangulación}

Como se mencionó anteriormente, en la investigación se entrevistó a profesores y profesoras, se observó una clase por cada profesor y profesora entrevistados, y se analizaron los temarios de esas clases. También se entrevistaron estudiantes. Es importante poner énfasis en todo lo anterior porque es la médula de este trabajo, debido a que todo tenía un propósito: constatar la intersubjetividad de las distintas informaciones vertidas por las distintas estrategias de investigación, información enraizada en diversas construcciones sociales que nos permitiera comparar interpretaciones, perspectivas y significados, confirmarlas o refutarlas. Este fue el ejercicio cualitativo de triangulación. La intención fue abarcar los mayores ángulos sociales posibles que permitieran realizar un análisis fidedigno y fundamentado. Por la misma razón se llevaron a cabo entrevistas con estudiantes de tercer año en adelante dentro del Programa de Trabajo Social, pues sus experiencias tomando clases con los(as) profesores(as), así como sus puntos de vista, sus percepciones y sus conocimientos representaban un elemento más de comparación, confirmación y contraste que aporta a la veracidad y la confiabilidad, tanto de los hallazgos como del análisis. 
Tal y como se tenía previsto, todo lo anterior permitió apreciar también las similitudes y contradicciones en los discursos y ejecutorias dentro de los salones de clase. Por ejemplo, hubo contradicción evidente en lo que dijeron los(as) profesores(as) acerca del abordaje de la temática de diversidad sexual entre los testimonios de las entrevistas, los comportamientos observados en sus clases y los postulados establecidos en los temarios de los cursos que ellos(as) ofrecen. La tendencia por parte de los(as) profesores(as) durante las entrevistas fue indicar que, cuando ofrecían sus cursos, siempre o con frecuencia, utilizaban ejemplos en relación a la temática de la diversidad sexual y de género. Sin embargo cuando se observaron sus ejecutorias en los cursos ofrecidos por estos y estas, fue patente que los ejemplos fueron escasos y en una clase inexistentes. No obstante, era de esperarse la escasez de ejemplos debido a que los temarios de las clases tampoco contemplaban la temática de la diversidad sexual. Por lo tanto, los(as) profesores(as) pueden tener conciencia sobre la importancia de incluir el tema de la diversidad sexual en sus cursos y testimoniar que tienen la intención para incluirlo, pero la realidad es que aun los currículos y los sílabos correspondientes continúan sin estar atemperados con la realidad social que se pretende abordar, lo que representa una gran limitación para las ejecutorias de los(as) profesores(as). Hay que entender que es muy difícil que los(as) profesores(as) escapen al contexto social y cultural en el que están inmersos(as) y donde fueron socializados(as), aunque lo cuestionen. También hay que entender que los discursos no se traducen totalmente en nuevas prácticas sociales.

A su vez, se encontró contradicción entre lo que dijeron los(as) profesores(as) y lo que dijeron los(as) estudiantes en torno a lo que hacen los(as) docentes, debido a que los(as) profesores(as) indicaron que cuando abordaban con ejemplos 
con relación a la temática, los(as) estudiantes se mostraban poco receptivos(as) y realizaban expresiones no verbales que reflejaban incomodidad. Sin embargo, los(as) estudiantes mencionaron que los(as) profesores(as), en su mayoría, no trabajan el tema. Incluso, un(a) estudiante señaló que los(as) profesores(as) tienden a tratar el tema como un tabú, como algo marginal. Un(a) estudiante respondió que cuando los(as) profesores(as) no dominan un tema, simplemente prefieren no trabajarlo o evadirlo, y creen que por eso no se trabaja el tema de la diversidad sexual. Con esto, apuntan también a la falta de conocimiento como una posible razón de la exclusión del tema de la diversidad sexual por parte de sus profesores(as). De esta misma manera, algunos de los(as) profesores(as) comentaron que son ellos(as) los que traen los ejemplos sobre diversidad sexual al salón de clases. No obstante, los(as) estudiantes contradijeron esa respuesta, ya que afirmaron que son ellos(as) quienes traen los ejemplos y las preguntas sobre el tema.

Esto pone de manifiesto una contradicción entre los discursos de ambas partes, que se podría vincular al desconocimiento sobre la temática y a la intolerancia que existe para abordar y trabajar temas como este, que para algunos sectores es un tabú o algo pecaminoso e irrelevante. Además, ambas partes indicaron que dentro del Programa de Trabajo Social existe una cantidad sustancial de personas (profesores(as) y estudiantes) fundamentalistas religiosos(as). Esto podría ser un elemento de deterioro o de obstáculo para el desarrollo e implementación del tema de diversidad sexual. Esta realidad evidencia la otra contradicción existente entre los propósitos establecidos en los Códigos de Ética de la Profesión con respecto a la justicia, las prácticas que se continúan reproduciendo en el interior del salón de clases en los procesos de enseñanza y aprendizaje, y los valores 
sociales prevalecientes en la sociedad puertorriqueña, con todos sus prejuicios y exclusiones.

Cuando se auscultaron sus opiniones en relación a si la profesión de Trabajo Social es heterosexista, los(as) estudiantes coincidieron con los(as) profesores(as). Ambos aclararon que el Trabajo Social es heterosexista debido al contexto en el cual está insertado y la conceptualización de las agencias y servicios. Hubo consenso entre los(as) estudiantes y profesores(as) al dar su opinión sobre si pensaban que la profesión de Trabajo Social era homofóbica, bifóbica y lesbofóbica. Ambos grupos expresaron que la homofobia, lesbofobia y bifobia venían de parte de ciertos(as) profesionales del Trabajo Social que lo reproducen, y no de la profesión como tal. No obstante, tanto profesores(as) como estudiantes coincidieron en que existe una relación entre el heterosexismo y la homofobia, lesbofobia y bifobia, ya que ambas tienen una finalidad o consecuencia negativa hacia las personas LHB, y la primera es causante de las demás. Es por esto que ese discurso se puede interpretar como una contradicción entre respuestas, ya que si el heterosexismo nutre a la homofobia, lesbofobia y bifobia, y todos(as) coincidieron en que la profesión es heterosexista, lo más lógico sería pensar que también es LHBfóbica. Se puede inferir que el problema subyace en el temor que existe a decir que la profesión es homofóbica, lesbofóbica y bifóbica porque sería como el equivalente de decir que es racista o clasista. Sin embargo, admitir que es heterosexista no lleva esa carga negativa tan grande.

La realidad es que el sol no puede taparse con una mano y todo se resume en un hecho bastante simple. Si el contexto es heterosexista porque valora y legitima exclusivamente la heterosexualidad, entonces es homofóbico, lesbofóbico y bifóbico porque claramente rechaza, minimiza, desvalida y 
oprime a las parejas del mismo sexo, y todo está fundamentado en ese heterosexismo excluyente de cualquier realidad social distinta.

Lo antes expuesto conduce a una conclusión bastante ineludible. Si el currículo no contempla de manera transversal la temática de la diversidad sexual, no se va a reflejar en los temarios ni en las clases. Si los sílabos no contemplan la diversidad sexual y los(as) profesores(as) que dictan los cursos fueron socializados(as) en el paradigma de la heterosexualidad, los(as) futuros(as) profesionales no se van a beneficiar del conocimiento del tema, no van a deconstruir normativas, bagajes y paradigmas discriminatorios, y saldrán al mundo a reproducir las mismas ideologías que mantienen invisibles, oprimidas y desventajadas a las personas LHB. La triangulación realizada demuestra la existencia de esta construcción social excluyente a través de los significados de los testimonios, comportamientos y los contextos curriculares que los enmarcan. Mientras la situación sea así, la temática queda a la discreción individual de los(as) profesores(as) y a sus niveles de conocimiento y experiencia en el mismo, que pueden ser amplios o pueden ser insuficientes. Esto tiene un impacto en la educación que reciben los(as) estudiantes de Trabajo Social, quienes en varias ocasiones expresaron no tener conocimiento, y tener dificultad para definir términos o para intervenir con personas LHB. Se puede afirmar entonces que el programa de clases, otrora la ética formal de la profesión en Trabajo Social, no prepara a futuros(as) profesionales del Trabajo Social capaces de intervenir justamente con las personas LHB.

La investigación demuestra que al terminar sus estudios, los(as) estudiantes de Trabajo Social no adquieren un conocimiento básico sobre diversidad sexual porque ni sus 
profesores(as) ni los temarios de los cursos proveen para ello. Los(as) estudiantes que tienen un conocimiento o interés básico sobre el tema de la diversidad sexual es porque en su mayoría tomaron un curso electivo sobre homosexualidad. Esto no es suficiente para preparar a los(as) futuros(as) profesionales de Trabajo Social para que cuestionen e intervengan con un tema tan complejo y que, a su vez, promuevan construcciones sociales de una humanidad más diversa y equitativa.

\section{Recomendaciones}

A raíz de todo lo estipulado anteriormente; de la información recopilada a través de diversas estrategias de investigación; del análisis de los hallazgos desde la perspectiva de un marco conceptual que incluye la perspectiva de género, el construccionismo social, la teoría crítica y la teoría de socialización; y la triangulación llevada a cabo con las informaciones y análisis resultantes, se desprenden varias recomendaciones que se entienden apremiantes para corregir el desfase educativo existente en torno a la temática de diversidad sexual y el desconocimiento generalizado sobre ese tema. Estas recomendaciones son formuladas en buena lid, con el mejor de los propósitos para mejorar la educación y la práctica de Trabajo Social.

A base de los hallazgos generados por las diversas estrategias investigativas, entendemos que se debe mejorar la educación en Trabajo Social, ya que en ninguna institución académica, sin importar la universidad o el nivel académico de la institución, se debe abordar marginalmente o como tabú el tema de la diversidad sexual y de género, ni se debe tratar con prejuicio o estigma a las personas LHB. Al contrario, 
la educación debe ser inclusiva, justa, equitativa y libre de prejuicios. Por tanto, se debe procurar educar en el campo de la diversidad sexual a los(as) estudiantes de Trabajo Social, que son las personas que serán los(as) futuros(as) profesionales que se insertarán en el campo laboral.

La realidad es que la profesión de Trabajo Social asume un discurso formal que se basa en los principios de la equidad, el respeto a la diversidad y la justicia social. Por tanto, cuando hablamos de una profesión cuya visión filosófica parte de estos tres conceptos, hay que entender que desde su génesis se debe encaminar hacia dicha visión. Pero la investigación evidencia que su discurso pedagógico se ajusta a esta visión solo parcialmente, es decir, que en su práctica no cumple con esas premisas. En fin, recibir una educación que excluye la diversidad sexual no propicia e impide el enriquecimiento intelectual, y no promueve el compromiso social de estos(as) estudiantes.

Un(a) profesional del Trabajo Social que se inserta en el mundo de la práctica directa o indirecta y que no reconoce la complejidad y la importancia de la diversidad sexual, podría ser devastador(a) porque se crearía un sesgo al intervenir con personas LHB o con temas de diversidad sexual. Ese sesgo no le va a permitir tener la oportunidad de romper con los constructos y visiones que se han internalizado y puede llevarlo(a) a estar prejuiciado contra los(as) participantes. Intervenir con personas LHB sin haber trabajado con ese sesgo podría desembocar en acciones que pudieran violar la ética de la profesión y la dignidad de cualquier ser humano.

Un(a) trabajador(a) social puede reflejar pensamientos heterosexistas, homofóbicos, lesbofóbicos y/o bifóbicos por diversas razones. Una de las razones de mayor peso es el desconocimiento y la ignorancia acerca de lo que es 
ser una persona lesbiana, homosexual o bisexual. Este desconocimiento podría ocasionar que haya profesionales faltando a la ética y que su comportamiento sea uno discriminatorio, lo que representaría una violación a los cánones de la profesión.

Es por todo lo anteriormente expuesto que se recomienda que se haga una revisión curricular profunda en los programas de Trabajo Social para atemperar los currículos e incluir la diversidad sexual y de género como uno de los temas que deben y tienen que abordarse. El tema de diversidad sexual debe estar de forma transversal en el currículo, de manera que todas las clases lo aborden y ese abordaje sea amplio, sea claro y permita que tanto los(as) profesores(as) como los(as) estudiantes amplíen su conocimiento sobre el mismo. Es imperativo integrar verdaderamente la diversidad sexual y de género en todos los cursos y temáticas, así como lo está la heterosexualidad. De este modo, no se requerirá una clase electiva y los(as) estudiantes se sentirán y estarán capacitados(as) para trabajar con personas LHB. Esto a largo plazo tendría un impacto en la profesión de Trabajo Social, pues a medida que se gradúen nuevos(as) profesionales, la práctica de la profesión debería transformarse poco a poco en una de verdadera inclusión que respete, proteja y luche por grupos oprimidos como lo son las personas LHB.

Debido a que el tema de diversidad sexual y de género es complejo, se recomienda al Colegio de Profesionales de Trabajo Social de Puerto Rico que promueva horas de educación continuada donde se les instruya a los(as) profesores(as) sobre el tema, de manera teórica y práctica. Eso ampliaría sus conocimientos y repercutiría en una práctica pedagógica efectiva que aborde de forma adecuada la temática de diversidad sexual. 
A los(as) profesores(as) se les hace una recomendación crítica de hacer una introspección; una autoevaluación que contemple qué tan instruidos e instruidas están verdaderamente en el tema de diversidad sexual. Se les insta a que evalúen cuáles son sus prejuicios y niveles de LHBfobias, pues podrían estar reproduciendo acciones discriminatorias de manera inconsciente. Además, se recomienda que revisen y amplíen sus conocimientos sobre la diversidad sexual y género, pues son los entes socializadores de los(as) estudiantes universitarios, lo que representa gran responsabilidad. Los(as) profesores(as) tienen el deber y la responsabilidad ética de conocer sobre el tema, romper con los esquemas y paradigmas arcaicos, y convertirse en vehículos para facilitar la inclusión de todos y todas. 


\section{Referencias}

Albite Vélez, Lilian (2004). El construccionismo social: Una mirada al desarrollo de esta perspectiva teórica en la sociología y la psicología social. Trabajo inédito para foro sobre construccionismo social y la investigación cualitativa. Universidad del Sagrado Corazón en San Juan, Puerto Rico.

Bogo, Marion, Daley, Andrea \& Newman, Peter (2009). Breaking the Silence: Sexual Orientation In Social Work Field Education. Journal of Social Work Education, Vol. (45), 7-27.

Borrillo, Daniel (2001). Homofobia. España, Barcelona: Ediciones Bellaterra.

Burgo, Nilsa (2011). Investigación cualitativa: miradas desde el Trabajo Social. Buenos Aires: Espacio Editorial.

Cain, Roy (1996). Heterosexism and Self-Disclosure in the Social Work Classroom. Journal of Social Work Education, Vol. 32 (1), 65-76.

Colegio de Profesionales del Trabajo Social de Puerto Rico (2011). Código de Ética Profesional. San Juan: CFM.

Freire, Paulo (1997). Pedagogía de la Autonomía. Enseñar exige respeto a la autonomía del ser del educando. Argentina: Siglo XXI Editores. Recuperado en www.slideshare.net/ Gatojazzy/paulo-freire-pedagoga-de-la-autonoma

Giddens, Anthony (2000). Sociología. [2ª Ed.] Madrid, España: Alianza Editorial. Recuperado en http://www.perio.unlp.edu. ar/catedras/system/files/anthony_giddens___sociologia.pdf

Giddens, Anthony (2003). Familia. Un mundo desbocado: Los efectos de la globalización en nuestras vidas (pp.65-79). 
[5 $5^{\mathrm{a}}$ Ed.] (P. Cifuentes Trad.) Madrid: Santillana Ediciones Generales.

Gorlier, Juna Carlos (2005). Construcción social, identidad, narración: nuevos enfoques teóricos y el (re) hacer del género. Argentina: Ediciones Al Margen.

Hutchins, Loraine \& Kaahumanu, Lani (1991). Bi any other name: Bisexual people speak out. Boston: Alyson.

Jayasingham, Dharman (2008). Knowledge/lgnorance and the Construction of Sexuality in Social Work Education. The Board of Social Work Education, Vol. 27(2), 138-151. doi: 10.1080/02615470701709469

Kisnerman, Natalio (2005). Pensar el trabajo social: Una introducción desde el construccionismo. [2 $2^{\mathrm{a}}$ Ed.] Buenos Aires: Lumen/Humanitas.

Lamas, Marta (1999) Género, diferencias de sexo y diferencia sexual. Recuperado en http://www.iupuebla.com/ Doctorado/Docto_Generoyderecho/ MA_Doctorado_ Genero/MA_GENERO.pdf.

Lamas, Marta (1995). La perspectiva de género. Revista de Educación y Cultura. Recuperado en http://www.latarea. com.mx/articu/articu8/lamas8.htm

Lance, Larry (2002). Heterosexism and Homophobia Among College Students. College Student Journal, Vol. 36 (3), 1-6.

Lucca, Nydia \& Berríos, Reinaldo (2009). La investigación cualitativa: fundamentos, diseños y estrategias. Cataño: Ediciones SM.

Maroto, Ángel (2006). Homosexualidad y Trabajo Social. Madrid: Consejo General de Diplomados Trabajo Social y Asistentes Sociales. 
Messinger, Lori (2004). Field Education in Social Work out in the field: Gay and lesbian social work students' experiences in field placement. Journal of Social Work Education Vol. 40 (2), 187-204. Recuperado en http://www.bu.edu/ssw/ files/2010/10/Breaking-the-silence- Sexual-orientation-infield-education1.pdf

Nieves, Luis (2011). Homofobia Trabajo Social y Políticas Públicas: ¿Dónde se intersecan la ética profesional y los valores religiosos? Hato Rey: Publicaciones Puertorriqueñas.

Parsons, Talcott (1951) El sistema social. El aprendizaje de las expectativas sociales de los roles y los mecanismos de socialización de la motivación. Recuperado en http://investigacion.politicas.unam.mx/ teoriasociologicaparatodos/pdf/Enfoque/Parsons\%20 -\%20El\%20sistema\%20social.pdf

Paul, Richard \& Elder, Linda (2003). La mini-guía para el pensamiento crítico: conceptos y herramientas. Fundación para el Pensamiento Crítico. Recuperado en http://www. criticalthinking.org/resources/PDF/ SP-ConceptsandTools. pdf

Puleo, Alicia (2007). Introducción al concepto de género. En Plaza J. \& Delgado, C. (Eds.) Género y Comunicación. España: Editorial Fundamentos.

Ritzer, George (1993). Teoría Sociológica Clásica. España: McGraw-Hill.

Sampieri, Roberto, Fernández, Carlos \& Batista, Pilar (2010). Metodologías de la investigación. [5 $5^{\mathrm{a}}$ Ed.] D.F., México: McGraw-Hill.

Serrano Francisca, Gómez, Antonio, Amat, Laura, \& López, Alexander (2012). Aproximación a la homofobia desde la 
perspectiva de los estudiantes de Educación Social de la Universidad de Murcia. Monográfico. Recuperado en http:// iesgtballester.juntaextremadura.net/web/profesores/tejuelo/ vinculos/articulos/mon06/05.pdf

Solé, Marçal \& Gredilla, Paso (2003). La lucha por la liberación gay y lesbiana. Puerto Rico: Organización Socialista Internacional.

Wisniewski, Jack \& Toomey, Beverly (1987). Are Social Workers Homophobic? Journal of the National Association of Social Workers, 32 (5), 454-455. 